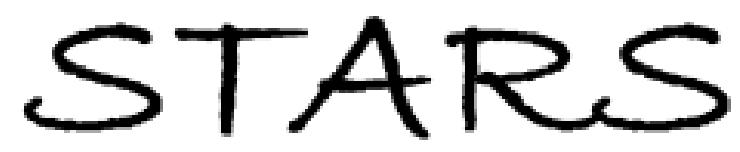

University of Central Florida

STARS

$1-1-2007$

\title{
Fabrication of nanogapped single-electron transistors for transport studies of individual single-molecule magnets
}

\author{
J. J. Henderson \\ University of Central Florida \\ C. M. Ramsey \\ University of Central Florida \\ E. del Barco \\ University of Central Florida
}
A. Mishra
G. Christou

Find similar works at: https://stars.library.ucf.edu/facultybib2000

University of Central Florida Libraries http://library.ucf.edu

This Article; Proceedings Paper is brought to you for free and open access by the Faculty Bibliography at STARS. It has been accepted for inclusion in Faculty Bibliography 2000s by an authorized administrator of STARS. For more information, please contact STARS@ucf.edu.

\section{Recommended Citation}

Henderson, J. J.; Ramsey, C. M.; del Barco, E.; Mishra, A.; and Christou, G., "Fabrication of nanogapped single-electron transistors for transport studies of individual single-molecule magnets" (2007). Faculty Bibliography 2000s. 7221.

https://stars.library.ucf.edu/facultybib2000/7221

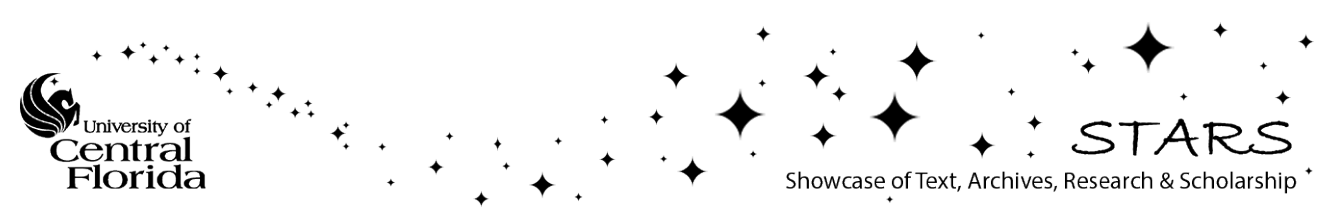




\section{Fabrication of nanogapped single-electron transistors for transport studies of individual single-molecule magnets}

Cite as: J. Appl. Phys. 101, 09E102 (2007); https://doi.org/10.1063/1.2671613

Submitted: 30 October 2006 . Accepted: 10 November 2006 . Published Online: 03 April 2007

J. J. Henderson, C. M. Ramsey, E. del Barco, A. Mishra, and G. Christou

\section{ARTICLES YOU MAY BE INTERESTED IN}

Fabrication of metallic electrodes with nanometer separation by electromigration Applied Physics Letters 75, 301 (1999); https://doi.org/10.1063/1.124354

The role of Joule heating in the formation of nanogaps by electromigration Journal of Applied Physics 99, 114316 (2006); https://doi.org/10.1063/1.2203410

Perspective: Thermal and thermoelectric transport in molecular junctions

The Journal of Chemical Physics 146, 092201 (2017); https://doi.org/10.1063/1.4976982

Lock-in Amplifiers up to $600 \mathrm{MHz}$

starting at $\$ 6,210$

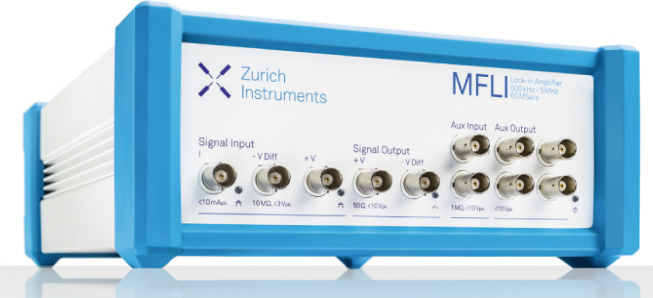

J. Appl. Phys. 101, $09 E 102$ (2007); https://doi.org/10.1063/1.2671613

(c) 2007 American Institute of Physics. 


\title{
Fabrication of nanogapped single-electron transistors for transport studies of individual single-molecule magnets
}

\author{
J. J. Henderson, C. M. Ramsey, and E. del Barco ${ }^{\text {a) }}$ \\ Department of Physics, University of Central Florida, 4000 Central Florida Boulevard, \\ Orlando, Florida 32816-2385
}

\author{
A. Mishra and G. Christou \\ Department of Chemistry, University of Florida, Gainesville, Florida 32611-7200
}

(Presented on 9 January 2007; received 30 October 2006; accepted 10 November 2006; published online 3 April 2007)

\begin{abstract}
Three-terminal single-electron transistor devices utilizing $\mathrm{Al} / \mathrm{Al}_{2} \mathrm{O}_{3}$ gate electrodes were developed for the study of electron transport through individual single-molecule magnets (SMMs). The devices were patterned via multiple layers of optical and electron beam lithography. Electromigration induced breaking of the nanowires reliably produces 1-3 nm gaps between which the SMM can be situated. Conductance through a single $\mathrm{Mn}_{12}$ (3-thiophenecarboxylate) displays the Coulomb blockade effect with several excitations within $\pm 40 \mathrm{meV}$. (c) 2007 American Institute of Physics. [DOI: $10.1063 / 1.2671613$ ]
\end{abstract}

\section{INTRODUCTION}

Electron transport properties of individual molecules have received considerable attention over the last several years due to the introduction of single-electron transistor (SET) devices, ${ }^{1-4}$ which allow the experimenter to probe electronic, vibrational, ${ }^{2}$ or magnetic ${ }^{3,4}$ excitations in an individual molecule. In a three-terminal molecular SET the molecule is situated between the source and drain leads with an insulated gate electrode underneath. The insulating ligands on the periphery of the molecule act as isolating barriers. Current can flow between the source and drain leads via a sequential tunneling process through the molecular charge levels, which the gate electrode is used to tune.

Conduction through a molecular SET only occurs when a molecular electronic level lies between the Fermi energies of the leads. A bias voltage $V_{\text {bias }}$ applied between the source and the drain changes the electrostatic potential of one of the leads by an energy $|e V|$. As the bias voltage is increased, excited states will provide conduction channels in the device and discrete changes in the current through the SET will be obtained every time a new molecular level falls within the bias window. Applying a gate voltage moves these molecular states with respect to the electrode Fermi levels.

Because the transport through single molecules proceeds via the discrete molecular levels, one can "spectroscopically" measure the quantum energy landscape of an individual molecule rather than an ensemble of molecules. This is particularly important for the single-molecule magnets (SMMs), where it has been shown that the molecule's crystalline environment has a profound influence on the spin Hamiltonian and therefore the quantum decoherence rates between $M_{S}$ levels.

Significantly quantum tunneling of the magnetization (QTM) can be observed between the different $M_{S}$ levels of

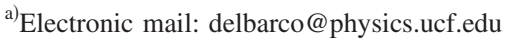

the molecule. ${ }^{4}$ This is a consequence of the quantum superposition of high-spin states of the molecule and has lead to the observations of a variety of fundamental phenomena, such as quantum (Berry phase) interference. ${ }^{6}$ Features of QTM are expected to manifest themselves in other observables as well. In particular, the effects of QTM on electronic transport remain to be explored in depth both experimentally and theoretically.

During the previous years, there has been a significant effort in this direction by both experimental and theoretical groups. Heersche et al. reported Coulomb blockade and conduction excitation characteristics of a molecular SET in an individual $\mathrm{Mn}_{12} \mathrm{SMM}$ functionalized with thiol groups. Negative differential conductance and current suppression effects were explained in terms of relaxation between excited spin levels of the charged and uncharged states of the $\mathrm{Mn}_{12}^{5}$. Jo et al. reported transport through an individual $\mathrm{Mn}_{12} \mathrm{SMM}$ without any functionalization of the molecules. They found evidence of magnetic anisotropy in some of the studied molecules. ${ }^{6}$ Even though these two results are very promising, they have failed to provide unambiguous evidence that transport occurred through an individual SMM that preserved key quantum properties found in their solid-state form (crystal). Several theoretical groups have proposed alternative ways to prove the SMM effect on the transport through a SET. ${ }^{7-11}$ Leuenberger and Mucciolo showed that QTM interference effects (Berry phase) can be tuned to modulate the Kondo effect in an isolated SMM-based SET. ${ }^{8}$ Similarly, Gonzalez and Leuenberger have recently shown that Berry phase effects can have a significant impact even in incoherent transport, away from the Kondo regime. ${ }^{11}$ These results indicate that Berry phase interference might be the most promising way to characterize the unique transport properties of SMMs in comparison with conventional, nonmagnetic molecules.

In this context, we have successfully fabricated threeterminal SET devices and prepared thiophenecarboxylate 


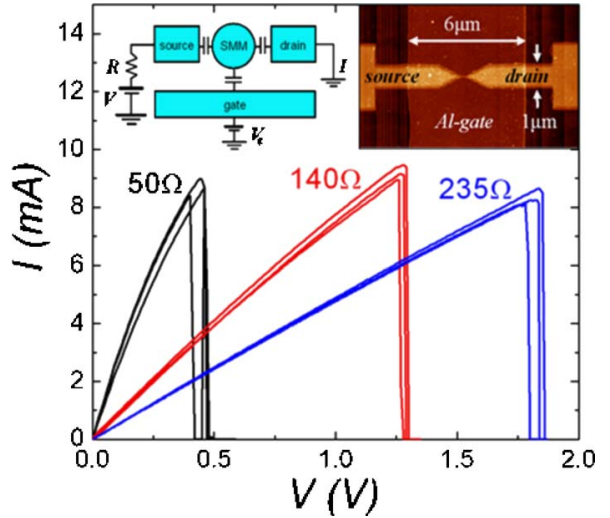

FIG. 1. (Color online) $I-V$ curves recorded during electromigration-induced wire breaking for three series resistances; left inset: Schematic representation of a three-terminal SET. Right inset: AFM image of a SET device.

functionalized $\mathrm{Mn}_{12}$ derivatives, which bind to $\mathrm{Au}$ surfaces in order to begin probing the transport properties of an isolated SMM. The compound of interest in the present study is $\mathrm{Mn}_{12}$ (3-thiophenecarboxylate). This is a derivative of $\mathrm{Mn}_{12}$ acetate for which the acetate group has been substituted with 3-thiophene carboxylate. The assembly of this molecule on $\mathrm{Au}$ and its bulk magnetic properties have previously been reported. $^{12}$ We have also synthesized the 2-thiophene carboxylate, 2-thiopheneacetate, and 3-thiopheneacetate variations for this study, but their results are not presented here.

In this report we present the technical aspects of our device fabrication and characterization as well as some preliminary results on transport through $\mathrm{Mn}_{12}$ thiophenecarboxylate).

\section{DEVICE FABRICATION}

Our SET devices are fabricated in a multistep process on high-quality silicon substrates coated with a flat $1 \mu \mathrm{m}$ thermal oxide layer. The recipe we follow is closely adapted from that of Ref. 13. Our devices are fabricated using three layers of optical lithography and one layer of e-beam lithography. In the first two steps, the contacts and leads to which nanowires are attached are patterned followed by evaporation of $\mathrm{Au}$ with $\mathrm{Cr}$ for adhesion. The first is a thin $30 \mathrm{~nm}$ layer of gold, which protrudes just beyond the $200 \mathrm{~nm}$ thick second layer leaving a step to which the nanowire will overlap. The thick $\mathrm{Au}$ evaporation for the second layer works to minimize the device resistance and provide a good surface for wire bonding. Next, the gate electrode is patterned and $\mathrm{Al}$ is evaporated while cooling the substrate with liquid $\mathrm{N}_{2}$. The gate is then allowed to oxidize overnight in atmospheric conditions, forming a $2-3 \mathrm{~nm} \mathrm{Al}_{2} \mathrm{O}_{3}$ layer. ${ }^{14}$ Finally, the nanowire pattern is written with electron beam lithography and gold deposited on that layer. An atomic force microscopy (AFM) micrograph of the completed device can be seen in Fig. 1 (right inset). At the narrowest part, the wire is $90 \mathrm{~nm}$ wide.

The nanometer size gap between the source and drain electrodes is formed by electromigration induced breaking of the nanowire. ${ }^{1}$ Figure 1 shows the electromigration induced breaking $(T=4 \mathrm{~K})$ of several wires fabricated following the process described above. The current through the nanowire is

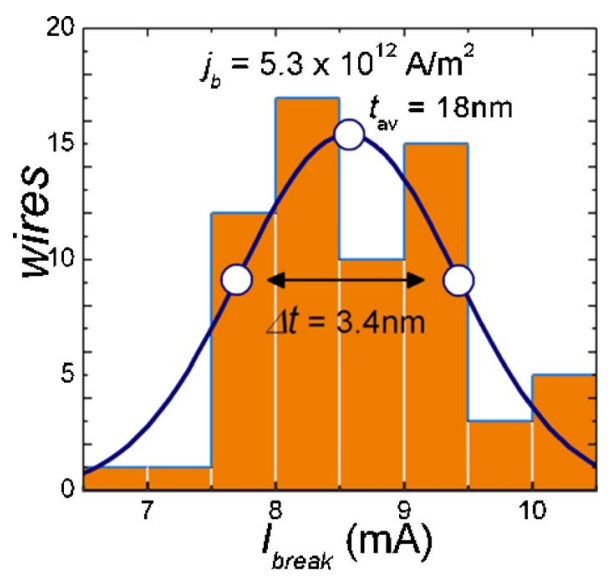

FIG. 2. (Color online) Distribution of breaking currents for a series resistance of $225 \Omega$. The distribution is associated with changes in the thickness $t$ of the gold nanowire.

essentially Ohmic up to a voltage at which the nanowire breaks $\left(V_{\text {break }}\right)$, producing a small gap. $V_{\text {break }}$ and thus the gap size are tunable by adding series resistors ${ }^{15}$ in line with the $30 \Omega$ nanowire as the different sets of data in Fig. 1 illustrate.

The size of the gap created by electromigration is mainly determined by the current density flowing through the nanowire. For a given current density, the higher $V_{\text {break }}$ the larger the size of the gap. In our devices, the postbreaking tunnel resistances vary between $10 \mathrm{k} \Omega$ and $100 \mathrm{G} \Omega$ for most of the wires. This huge variation corresponds to only an $\sim 1 \mathrm{~nm}$ variation in gap size according to previous studies. ${ }^{13}$ Thus our gap sizes are generally in the $1-3 \mathrm{~nm}$ range. The critical current density necessary to break a gold nanowire is estimated to be $j_{b}=5 \times 10^{12} \mathrm{~A} / \mathrm{m}^{2}$ (Ref. 15) and the breaking current $I_{\text {break }}$ necessary to achieve this characteristic current density depends on the cross sectional area of the nanowire. Therefore, $V_{\text {break }}$ is determined by the total resistance of the circuit as follows: $V_{\text {break }}=R_{\text {total }} I_{\text {break }}$, where $R_{T}=R_{\text {nanowire }}$ $+R_{\text {series }}$. The histogram in Fig. 2 shows that the distribution of $I_{\text {break }}$, as measured from the electromigration data, is centered at $8.5 \mathrm{~mA}$. Considering a cross sectional area of $90 \mathrm{~nm}$ (width) $\times 18 \mathrm{~nm}$ (thickness) for our nanowires, we obtain $j_{b}=5.3 \times 10^{12} \mathrm{~A} / \mathrm{m}^{2}$, which is in excellent agreement with the accepted value. ${ }^{15}$ If we associate the change in breaking currents $\left(\Delta I_{\text {break }} \sim 2 \mathrm{~mA}\right)$ to variations of the nanowire thickness, this gives us a $3.4 \mathrm{~nm}$ thickness variation along the 3 in. length of the silicon wafer used in fabrication. This variation is due to dispersion of the gold evaporation beam along the wafer (solid angle), since each point of the wafer is not equidistant from the source.

Figure 3 shows four different characteristic $I-V$ curves measured directly after electromigration induced breaking of the nanowire. The inset summarizes the percentage for which we observe each of the various curves. Here, we neglect to show the nonconducting curves, which occur about $50 \%$ of the time due to large $(>3-4 \mathrm{~nm})$ gap formation. The $I-V$ characteristic curves of broken wires can then be grouped together as follows: ${ }^{16}$ (a) CB curves with current suppression for low bias voltages consistent with Coulomb blockade effect; (b) STP, curves showing abrupt changes of current 


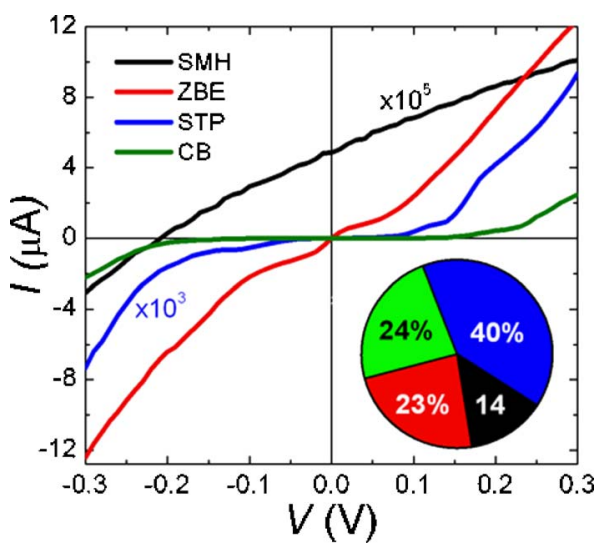

FIG. 3. (Color online) Selection of different $I-V$ curves observed after wire breaking: asymmetric smooth curves (SMH), curves with zero bias enhancement of the current (ZBE), curves with steps (STP), and curves with current suppression at low voltages consistent with Coulomb blockade (CB): Inset: Pie chart showing statistics of the $I$ - $V$ curve types found after the breaking of over 60 nanowires.

(steps) consistent with Coulomb blockade effect in molecular SETs; (c) ZBE, zero bias enhancement of the conductance consistent with low temperature Kondo effect; and (d) $\mathrm{SMH}$, smooth asymmetric curves not crossing $I=V=0$. The curves shown occur on different current scales ranging from microampere down to tens of picoampere but are normalized to microampere for ease in comparison of their features.

\section{SINGLE-MOLECULE TRANSPORT MEASUREMENTS}

The $\mathrm{Mn}_{12}$ (3-thiophenecarboxylayte) for SET studies was prepared by dissolving a polycrystalline sample in $\mathrm{CH}_{2} \mathrm{Cl}_{2}(0.1 \mathrm{mM})$. The devices were cleaned by oxygen plasma and immediately immersed into the solution for about $90 \mathrm{~min}$. After the allotted time, the sample was removed from the solution, rinsed with $\mathrm{CH}_{2} \mathrm{Cl}_{2}$, and blown dry with a stream of $\mathrm{N}_{2}$. Following self-assembly of the molecules, electromigration breaking and measurements were performed at $4 \mathrm{~K}$.

Figure 4 displays the differential conductance $(d I / d V)$ contour plotted as a function of the bias and gate voltages (the added lines are to help display the linear conductance excitations present in this particular measurement). The presence of multiple parallel excitations forming the characteristic diamond shape of a molecular SET indicates the crossing between two charge states $(N$ and $N+1)$ of the molecule and reveals the complex nature of the charge states of this particular molecule. The excitations are found in an energy range $(0 \pm 40 \mathrm{meV})$ similar to what has been observed by other authors in $\mathrm{Mn}_{12}$-based SETs. ${ }^{5,6}$ We have observed several other molecules behaving similarly in experiments carried out at $4 \mathrm{~K}$ in the same set of devices; however, many of the molecules measured were not stable enough to measure for long periods of time. Out of 60 electromigration-broken wires, we have seen 3 that were stable enough to measure.

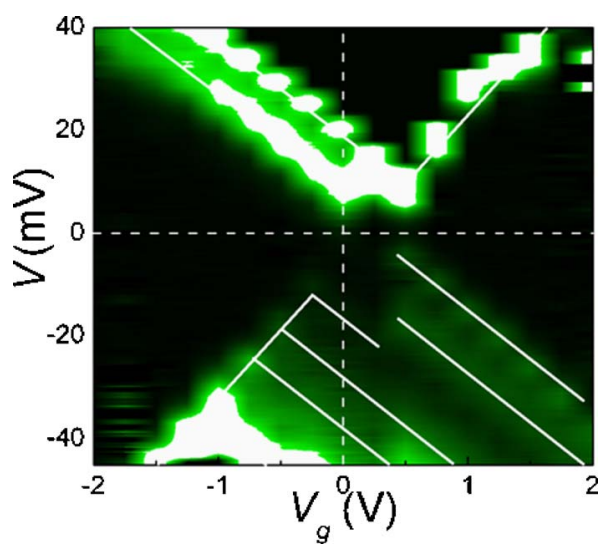

FIG. 4. (Color online) Conductance $(d I / d V)$ contour plot as a function of the bias and gate voltages, showing the typical Coulomb diamond response of a molecular SET. Interestingly, at least seven excited states are observed.

\section{CONCLUSIONS}

We have outlined the technical details of our singleelectron transistor devices and demonstrated that they can be used to measure single-electron transport through individual $\mathrm{Mn}_{12}$ SMMs. Our current objective is to probe different SMMs at low temperatures $(>15 \mathrm{mK})$ and in the presence of high magnetic fields generated by a vector superconducting magnet which allows arbitrary orientation of the magnetic field with respect to the geometry of the SMM-based SET.

\section{ACKNOWLEDGMENT}

One of the authors (J.J.H.) thanks the $\mathrm{I}^{2} \mathrm{Lab}$ at UCF for financial support.

${ }^{1}$ H. Park, A. K. L. Lim, A. P. Alivisatos, J. Park, and P. L. McEuen, Appl. Phys. Lett. 75, 301 (1999).

${ }^{2}$ H. Park, J. Park, A. K. L. Lim, E. H. Anderson, A. P. Alivisatos, and P. L. McEuen, Nature (London) 407, 57 (2000).

${ }^{3}$ J. Park et al., Nature (London) 417, 722 (2002).

${ }^{4}$ J. R. Friedman, M. P. Sarachik, J. Tejada, and R. Ziolo, Phys. Rev. Lett. 76, 3830 (1996).

${ }^{5}$ H. B. Heersche, Z. De Groot, J. A. Folk, and H. S. J. van der Zant, Phys. Rev. Lett. 96, 206801 (2006).

${ }^{6}$ M.-H. Jo et al., Nano Lett. 6, 2014 (2006).

${ }^{7}$ C. Romeike, M. R. Wegewijs, W. Hofstetter, and H. Schoeller, Phys. Rev. Lett. 96, 196601 (2006).

${ }^{8}$ M. N. Leuenberger and E. R. Mucciolo, Phys. Rev. Lett. 97, 126601 (2006).

${ }^{9}$ C. Romeike, M. R. Wegewijs, and H. Schoeller, Phys. Rev. Lett. 96, 196805 (2006).

${ }^{10}$ C. Romeike, M. R. Wegewijs, W. Hofstetter, and H. Schoeller, Phys. Rev. Lett. 96, 196601 (2006)

${ }^{11}$ G. Gonzalez and M. N. Leuenberger, e-print cond-mat/0610653.

${ }^{12}$ B. J. Kim, B. J. Suh, S. Yoon, S. Phark, Z. G. Khim, J. Kim, J. M. Lim, and Y. Do, J. Korean Phys. Soc. 45, 1593 (2004); S. Phark, Z. G. Khim, B. J. Kim, B. J. Suh, and S. Yoon, Jpn. J. Appl. Phys., Part 1 43, 8273 (2004).

${ }^{13}$ J. Park, Ph.D. thesis, University of California-Berkeley, 2003.

${ }^{14}$ S. H. Magnus Persson and L. Ollofsson, Appl. Phys. Lett. 74, 2546 (1999).

${ }^{15}$ C. Durkan, M. A. Schneider, and M. E. Weilland, J. Appl. Phys. 86, 1280 (1999).

${ }^{16}$ L. H. Yu and D. Natelson, Nanotechnology 15, S517 (2004); L. H. Yu, Z. K. Keane, J. W. Ciszek, L. Cheng, M. P. Stewart, J. M. Tour, and D. Natelson, Phys. Rev. Lett. 93, 266802 (2004); H. S. J. van der Zant et al., Faraday Discuss. 131, 347 (2006). 